\title{
Visual Search Behaviour amongst Arabic, English and Chinese Search Engine Users
}

\author{
Mohammad Alsaffar, Lyn Pemberton, Karina Rodriguez Echavarria \\ University of Brighton, UK \\ Email: M.Alsaffar@brighton.ac.uk \\ Email:Lyn.Pemberton@brighton.ac.uk \\ Email: K.Rodriguez@brighton.ac.uk
}

\begin{abstract}
Human Computer Interaction $(\mathrm{HCl})$, the discipline established to analyse interactions between users and their technologies, encompasses the study of the effectiveness of the results provided by the search engines that are such a vital part of current web use. Although search engine technologies are used across the globe, studies focusing on how results are viewed by regionally diversified users are scarce. The aim of this research is to investigate possible variations in visual search behaviour and eye movements on Search Engine Results Pages (SERPs) among three user groups (Arabic speakers, English speakers and Chinese speakers). This study contributes to the field of Web Design and search behaviour by providing evidence of the similarities and differences between users from the Middle East, the West and the Far East, from the perspective of visual search behaviour and eye movement.
\end{abstract}

Visual search behaviour, SERP, Eye tracking, Arab, English, Chinese.

\section{INTRODUCTION}

The dramatic growth in the number of websites available for users has made website designers aware of the need to use components of web design including colours, images and layout effectively to attract and retain the attention of web site users across the globe (Reinecke et al. 2014). Both website authors and end-users can benefit by establishing an understanding of which elements of web pages attract most visual attention (Prisacari \& Holme 2013). Search engines are a very popular and important type of website, used by a global audience. Searching for information effectively on the internet is becoming an unavoidable part of daily life tasks such as communicating, learning and shopping. Search engines act as a gateway to the world of endless websites and help users find websites relevant to their interests and needs. Interpretation and measurement of the visual search behaviour of users, including those with different cultural and language backgrounds, could enable modern search engines to personalise and customise results based on these differences. Moreover, there may be important academic and commercial implications in better understanding how users search for information in Search Engine Results Pages (SERPs). The results of this comparative investigation into the visual search behaviour of culturally and regionally diversified users of search engines results pages have the potential to enable companies to formulate strategies to attract more global users to their websites.

Very little attention has been paid to the visual processing of users of different culture and language. Arabic, English and Chinese people differ in terms of culture, language and aspects of cognition. For example, significant differences were found between Chinese and Western European users' online search behaviour when they were examined for type of information sought, frequency, goal, usage pattern and websites selected in a cross-cultural comparative study (Vuylsteke et al. 2009). However, this study was based only on interviews and questionnaires. It has also been noted that Arabic users exhibited differences in their visual patterns while interacting with Arabic interfaces comparing to those patterns on English interfaces with English users (George et al. 2011). These differences might have an influence on visual behaviour and this was the motivation behind the present study.

For the purpose of this study, the Google SERP interface was selected since it is by far the highest used search engine in the world (NetMarketShare 2017). The participants were exposed to SERP interfaces from Google with three different presentations. As shown in Figure 1, the first is a Plain PC SERP, i.e. a list of results without images, rich snippets and elements on the right hand side. 
The second the Rich PC SERP with images, rich snippets and elements of the right hand side. The third is a Mobile SERP.

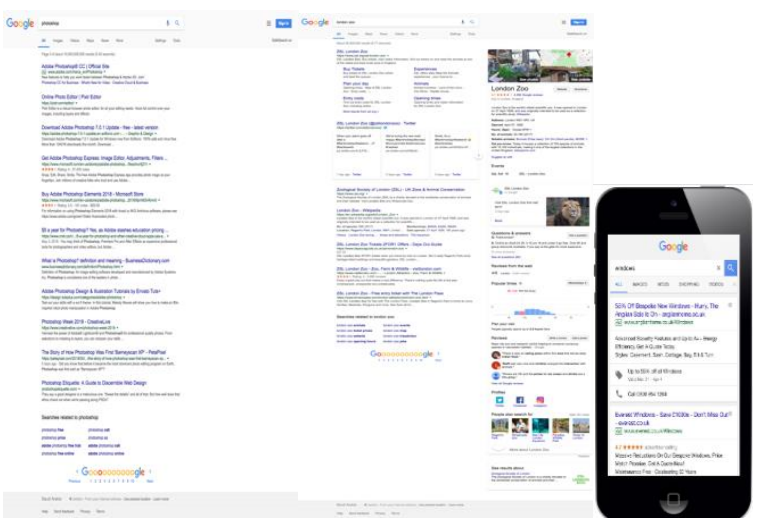

Figure 1: Examples of SERP types

The purpose of such diversity is to cover a variety of SERPs that users might find in different search engines and to elicit the potential differences in their performance and preferences. Web designers can benefit from understanding the visual search behaviour of search engine users, as exposed using eye-tracking (Cutrell \& Guan 2007). This study investigates the visual behaviour of three culturally diverse groups of users when searching the SERPs. It aims to address the question "Do Arabic, English and Chinese users differ in their visual search behaviour when using the same English Search Engine?"

For decades, eye tracking technology has been used to explore non-digital human activities such as reading, scanning and processing of visual stimuli (Sharma \& Dubey 2014). Eye tracking can also be a very effective tool to study users' behaviours during online information processing, including using search engines. Marketing companies, usability specialists and research scientists have used eye-tracking technology extensively in online research (Granka et al. 2008).

The significance of this research is to determine if users' cultural and language background differences require local adaptation to produce search engine result pages to match users' expectations and searching strategies. In particular, since user groups selected for this study have different reading directions, English and Chinese being read left-toright and Arabic right-to-left), it is interesting to explore these groups' visual search behaviour and to measure the possible impact of these differences on the cognitive process of information. In addition, many studies suggest that the different writing systems used to represent different languages have an impact on the way information is processed by individuals (Schmitt et al. 1994). Most current search engines interfaces are almost identical for global users disregarding their country or background (Marcos et al. 2013) and there is a lack of studies to investigate how non-English speakers interact with SERPs presenting results in English. Additionally, since the advent of smartphones and their growing use globally, a significant amount of web browsing and searching activities has been shifted from desktops to smartphones (Kim et al. 2015). Few empirical studies have analysed the processing of web results on smartphones screens using eye tracking technologies, hence the inclusion of a mobile SERP in this study.

The rest of the paper is organised as follows. Section 2 describes the methodology employed in the study. Section 3 presents the results obtained from the experiment with discussion.

\section{METHODOLOGY AND EXPERIMENT DESIGN}

Eye-tracking Tobii Pro X2 was used to record eye movements of subjects as the primary step of data collection. Objective data such as visual search behaviour was gathered using the eye-tracking system. Researchers can separate different elements of a visual stimulus into "areas of interest" (AOl's) such as top banner, bottom banner etc. for the sake of statistical analysis. Eye-fixation is generally considered to be the most relevant indicator to evaluate and process information acquisition. Other eye movement metrics, consistent with the other studies (Jacob \& Karn 2003; Pan et al. 2004), have been used in this study. These are total fixation duration and dwell time, represented in heat maps. Total fixation duration evaluates the total duration of participants' fixation on a specific visual stimulus, while the average fixation duration indicates cognitive processing. A heat map is a visual presentation based on the fixation duration of participants' attention of SERP pages where red spots reflect most time fixated while the green and yellow show the lower fixated. Heat maps are more useful when they are generated by the aggregated data collected from a number of participants to provide combined results.

Overall, 90 participants took part in this study: 30 Arabs, 30 English and 30 Chinese national participants. All participants were undergraduate or postgraduate students from the University of Brighton. This ensured that the participant had a minimum level of English proficiency required for this study.

The search engine results page were real pages taken from the Google search engine. Three types of search engine results pages were developed: two for the desktop large screens and one for the small screen (i.e. smartphone). Each participant carried out three tasks for each type of SERP. All tasks were from general domains without specific topics, aiming to reduce the familiarity of those tasks, generalise the findings and trying to counterbalance across participants. 
Each SERP tested was divided into three main sections of the Google page: Ads section, Top section and Bottom section. The fixation duration of the three tasks performed in each SERP was combined to represent the total fixation duration of the tasks. For example, to have the total fixation duration of the Ads section in the plain PC SERP, the fixation duration of the Ads AOls for each group in the three tasks was combined using the sum function in SPSS. As shown in Figure 2, the Ads section represents the advertisement results that usually appears to the users on the top. The Top section includes the first three organic results that located on the top of the presented SERP. The Bottom section represents the last three organic results of the SERP. In the Rich PC SERP, as this type of SERP has only the elements of the right hand side: therefore, an extra part was added which represents the right hand side section in the three tasks. A $3 \times 3$ mixed design ANOVA test was conducted on each of the identified sections of each SERP to examine any significant impact of SERP type and group type in the fixation duration in the four sections (Ads, Top, Bottom and Right).

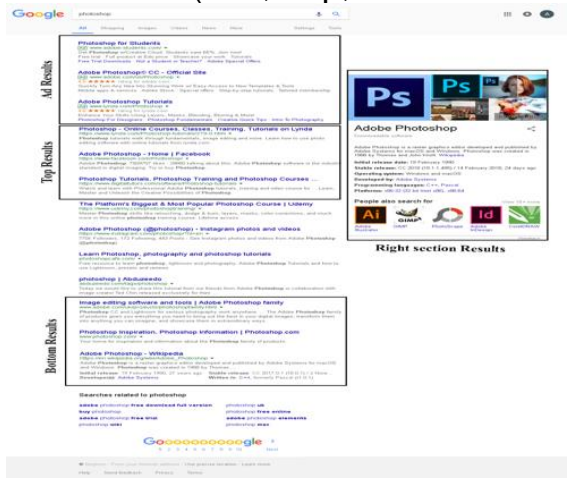

Figure 2: Different Sections of SERP

\section{EXPERIMENTAL RESULTS AND DISCUSSION}

In the following subsections, two types of analysed data are presented. Sections 3.1 and 3.2 show differences in fixation duration in the different sections of the page across the three groups, while section 3.3 shows the participants' fixation duration distributions using heatmaps.

\subsection{Fixation Duration in the Ads, Top and Bottom Sections across the SERP Presentation Types}

The $3 \times 3$ mixed design ANOVA test revealed a significant main effect of SERP type on fixation duration on Ads section $F(2,174)=84.94, p<0.001$, Top section $F(2,174)=18.57, p<0.001$ and Bottom section $F(2,152)=84.94, p<0.001$. An inspection of the mean scores indicated that the average total fixation duration in Top section in all SERPs received the highest fixation duration from all groups $(M=14.83 \mathrm{sec}, S D=7.41)$ compared to Ads section $(M=12.97 \mathrm{sec}, S D=7.92)$ and the Bottom section were less than the Top and Ads sections by at least $50 \%(M=7.2 \mathrm{sec}, S D=5.12)$. More specifically, in Ads section, the Ads results in the mobile SERP received the longest fixation duration from all groups $(M=19.55 \mathrm{sec}, S D=10.91)$ followed by the Ads section in the rich PC SERP $(M=10, S D=6.28)$. The shortest fixation duration was on the plain PC SERP Ads section with $(M=9.11, S D=6.57)$. In terms of Top section, the top results in the rich PC SERP were most viewed $(M=17.23, S D=8.86)$ followed by mobile SERP $(M=16.01, S D=7.7)$ and the plain SERP was the lowest $(M=11.25, S D=$ 5.69). Lastly, for the Bottom section, the results that located at the bottom in the rich SERP were fixated more $(M=9.92, S D=7.27)$ than those located in the plain SERP $(M=5.13, S D=3.74)$ and mobile SERP $(M=6.55, S D=4.37)$. In addition, the type of the group had a significant main effect on fixation duration on Ads section $F(2,87)=22.66, p<0.001$, Top section $F(2,174)=17.33, p<0.001$ and Bottom section $F(2,76)=41.60, p=0.001$.

English participants in all sections in each SERP had the lowest fixation duration comparing to Arab and Chinese. Note the Arabs' fixation duration on the Ads section and Top section in all SERPs is almost twice the English speaker fixation duration in those sections. However, the post hoc Pairwise Comparisons indicated exactly where the significances appear between the three groups in each section for each SERP type. Starting with Ads section, English users had significantly $(p<0.001)$ lower fixation duration on the Ads section in all SERP types with $(M=7.94, S D=4.78)$ compared to Arabs $(M=17.01, S D=8.65)$ and to Chinese $(M$ $=13.95, S D=6.85)$ except in rich SERP $(p>0.05)$. There was no significant difference between Arab and Chinese users in fixation duration on $A d s$ section in the plain and mobile SERP $(p>0.05)$ and were only significant higher than Chinese fixation duration in the rich PC SERP $(p<0.001)$.

Chinese users fixated almost the same time on the Ads section in the plain PC SERP and the rich PC SERP. Regarding Top section, English users significantly $(p<0.05)$ fixated on Top section in all SERPs $(M=10, S D=5.16)$ less than Arab $(M=$ $17.51, S D=9.79)$ and Chinese $(M=16.97, S D=$ 7.30). No significant differences were found between Arab and Chinese ( $p>0.05)$. Lastly, in the Bottom section, significant differences between groups were found only in the mobile SERP. English users had significantly $(p<0.001)$ lower fixation duration on bottom results $(M=3.46, S D=$ 2.43) compared to Arab $(M=8.04, S D=6.25)$ and Chinese $(M=8.15, S D=4.43)$. No significant differences were found between the three groups in the plain and rich SERP $(p>0.05)$. Finally, the interaction between group type and SERP type was significant only in Ads section $F(4,174)=5.37$, $p<0.001$. In Top and Bottom sections no significant interaction was found $(p>0.05)$. 


\subsection{Fixation Duration in the Right Section across the Three Tasks in Rich PC SERP}

AOls in the three tasks performed in the rich PC SERP that represented the elements on the right hand side were combined and the fixation duration were collected from all participants. An independent one Way ANOVA test was conducted to compare the effect of group type (Arab, English and Chinese) on fixation duration in Right section. There was a significant main effect of group type on fixation duration at the $p<0.05$ level for the three groups $[F$ $(2,83)=7.23, p=0.001]$. Post hoc comparisons using the Bonferroni HSD test indicated that the mean fixation duration for English $(M=12.61 \mathrm{sec}$, $S D=6.52)$ were significantly lower fixation duration than $\operatorname{Arab}(M=21.08 \mathrm{sec}, S D=12.74)$ and Chinese $(M=20.18 \mathrm{sec}, S D=7.47)$. However, Arab and Chinese did not significantly differ from each other in their fixation duration ( $p>0.05)$.

From the results above generally English always had the lowest fixation duration in all sections across all SERPs followed by Chinese users in all tasks, and the Arab users made the longest duration of fixations. Moreover, It obvious that Arab fixated more on the Right section than English and Chinese users. A possible explanation for these results may be the language impact. The language direction might make the searching easier for English and Chinese as they used to read from the left-to-right, while Arab users, even though they have high skills in reading English sentences, they made a great deal of fixation duration.

The top results were viewed more than the bottom results, and that was in line with (Guan \& Cutrell 2007) study. The ad results in the mobile SERP were received more fixations and attention from all groups compared to the ad results those presented in the PC SERP whether the plain or the rich PC SERP. This gives an indication of the importance of ads results on the Google mobile search. Moreover, it has been argued that many users exhibit Ads blindness while interacting with webpages (Pagendarm \& Schaumburg 2006; Owens et al. 2011) or try to ignore ads on SERPs (Alsaffar et al. 2017). However, in this study, ad results especially in the mobile SERP received a substantial amount of attention specifically by Arabs followed by Chinese users, which propose encouraging findings to international marketing sectors.

\subsection{Heatmaps}

Figures 3 and 4 show heatmaps that represent the aggregated fixation duration from all Arabic, English and Chinese participants in the two PC SERP presentations (Plain PC SERP and Rich PC SERP). Mobile heatmaps share the similar patterns and were not included due to the page limitations.

Clearly, Arab and Chinese tended to read the ad results more intensively comparing to English. Arab users read the result from the beginning of the result until the end in all results especially in the plain SERP, even though the first two or three results are advertisements. English users tended to jump to the next result without reading the whole sentence and tended to ignore the first two results, as they were advertisements. Moreover, interestingly English did not read from the beginning of the result listed and they more started from the middle of the result sentence trying to grab the keyword that answer the search question. Regarding Chinese users, their eye movements scattered around the SERP. They tended to read each result but without a connection of their fixations. There were white spots between each fixation in a single line. In conclusion, Arab fixated most often on the beginning of each sentence, English fixated most often on the middle and Chinese scattered their fixations around the page.

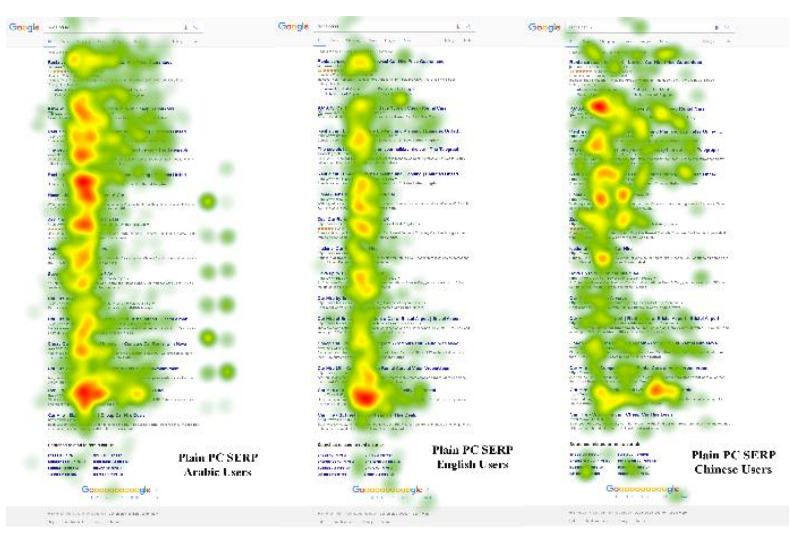

Figure 3: Arabic, English and Chinese heatmaps on Plain PC SERP

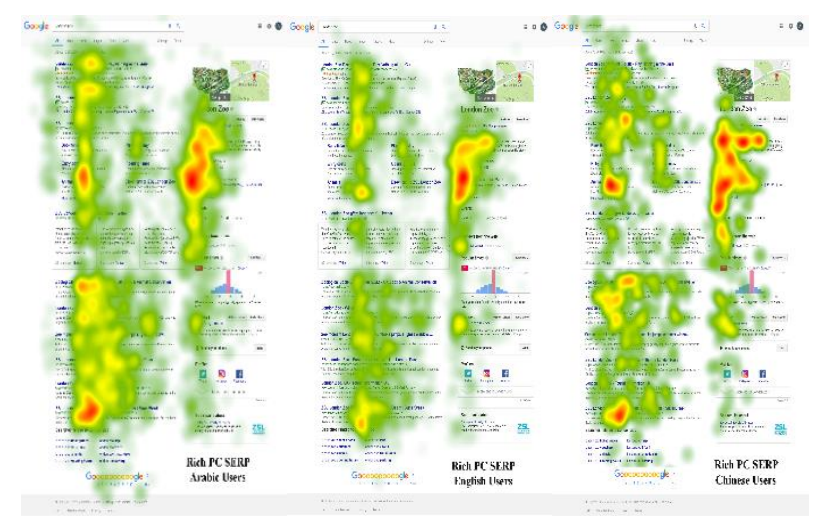

Figure 4: Arabic, English and Chinese heatmaps on Rich PC SERP

\section{FUTURE DIRECTIONS}

In future work, it is possible to give the participants the freedom in producing their own queries. A further extension would be also to analyse the performance of users and whether the language direction has an influence on their success rate. Users preferences of the SERP type would also be interesting to find. 


\section{REFERENCES}

Alsaffar, M. et al., 2017. Visual behaviour in searching information: A preliminary eye tracking study. In 2017 11th International Conference on Research Challenges in Information Science (RCIS). IEEE, pp. 365370.

Cutrell, E. \& Guan, Z., 2007. What are you looking for? An eye-tracking study of information usage in web search. In Proceedings of the 25th SIGCHI Conference on Human Factors in Computing Systems. San Jose, California, USA, pp. 407-416.

George, R.P., Anwar, R. \& Jeyasekhar, S., 2011. Visual Reading Patterns on Arabic Interfaces: Insights from Eye Tracking. Journal of Computing, 3(11), pp.109-114.

Granka, L., Feusner, M. \& Lorigo, L., 2008. Eye Monitoring in Online Search. Passive Eye Monitoring, pp.347-372.

Guan, Z. \& Cutrell, E., 2007. An Eye Tracking Study of the Effect of Target Rank on Web Search. In Proceedings of the 25th SIGCHI Conference on Human Factors in Computing Systems. San Jose, California, USA: ACM, pp. 417-420.

Jacob, R.J.K. \& Karn, K.S., 2003. Eye tracking in Human-Computer interaction and usability research: Ready to deliver the promises. In The Mind's Eye: Cognitive and Applied Aspects of Eye Movement Research. Oxford: Elsevier Science BV., pp. 573-605.

Kim, J. et al., 2015. Eye-Tracking Analysis of User Behavior and Performance in Web Search on Large and Small Screens. Journal of The Association For Information Science and Technology, 66(3), pp.526-544.

Marcos, M. et al., 2013. Cultural Differences on Seeking Information: An Eye Tracking Study. In CHl' 13. Paris, France: ACM.

NetMarketShare, 2017. Market Share Statistics for Internet Technologies. Available at: https://netmarketshare.com/search-enginemarket-share.aspx [Accessed May 14, 2018].

Owens, J.W., Chaparro, B.S. \& Palmer, E.M., 2011. Text Advertising Blindness: The New Banner Blindness? Journal of Usability Studies, 6(3), pp.172-197.

Pagendarm, M. \& Schaumburg, H., 2006. Why Are Users Banner-Blind? The Impact of Navigation Style on the Perception of Web Banners. Journal of Digital Information, 2(1).

Pan, B. et al., 2004. The determinants of web page viewing behavior: an eye-tracking study. Proceedings of the ETRA '04 Symposium on Eye Tracking Research and Applications, 1(212), pp.147-154.

Prisacari, A. \& Holme, T., 2013. Using EyeTracking to Test and Improve Website Design. In Design, User Experience, and
Usability. Design Philosophy, Methods, and Tools. Springer Berlin Heidelberg, pp. 389398.

Reinecke, K., Arbor, A. \& Gajos, K.Z., 2014. Quantifying Visual Preferences Around the World. In Proceedings of the SIGCHI Conference on Human Factors in Computing Systems. Toronto, ON, Canada: ACM, pp. 11-20.

Schmitt, B.H., Pan, Y. \& Tavassoli, N.T., 1994. Language and Consumer Memory: The Impact of Linguistic Differences between Chinese and English. Journal of Consumer Research, 21, pp.419-431.

Sharma, C. \& Dubey, S.K., 2014. Analysis of Eye Tracking Techniques in Usability and $\mathrm{HCl}$ Perspective. In International Conference on Computing for Sustainable Global Development (INDIACom). pp. 607-612.

Vuylsteke, A. et al., 2009. Consumers ' Online Information Search : A Cross-Cultural Study between China and Western Europe. Business, pp.1-34. 